BULL. AUSTRAL. MATH. SOC.

VOL. 34 (1986) 411-413.

\title{
A THEOREM ON COMMUTATIVITY
}

\author{
OF SEMI-PRIME RINGS
}

\section{Murtaza A. Quadri and Mohd. Ashraf}

The following Theorem is proved: Let $R$ be a semi-prime ring in which either $(x y)^{n}-x^{n} y^{n}$ or $(x y)^{n}-y^{n} x^{n}$ is central, for all $x, y$ in $R$ where $n>1$ is a fixed integer. Then $R$ is commutative.

\section{Introduction.}

A theorem of Herstein [6] states that a ring $R$ satisfying the identity $(x y)^{n}=x^{n} y^{n}$, where $n>1$ is a fixed positive integer, must have nil commutator ideal. Later Awtar [3] and Abu-Khuzam [1] established commutativity of the rings satisfying the above identity imposing the torsion conditions on the additive group $R^{+}$. In this direction Bell [5] proved that if $R$ is an $n$-torsion free ring with identity 1 and satisfies the two identities $(x y)^{n}=x^{n} y^{n}$ and $(x y)^{n+1}=x^{n+1} y^{n+1}$, then $R$ is commutative. Recently Abu-Khuzam [2] extended the mentioned results as follows: "If $R$ is a semi-prime ring in which, for each $x$ in $R$, there exists an integer $n=n(x)>1$ such that $(x y)^{n}=x^{n} y^{n}$, for all $y$ in $R$, then $R$ is commutative."

Received 6th January 1986.

Copyright Clearance Centre, Inc. Serial-fee code: 0004-9727/86 $\$ A 2.00+0.00$. 
our present aim is to generalize the above result. In fact we prove the following:

THEOREM: Let $n>1$ be a fixed positive integer and $R$ be a semiprime ring which satisfies one of the following polynomial identities:

$$
\begin{aligned}
& \left(z_{1}\right) \text { For alz } x, y, z \text { in } R,\left[(x y)^{n}-x^{n} y^{n}, z\right]=0, \\
& \left(z_{2}\right) \text { For alz } x, y, z \text { in } R,\left[(x y)^{n}-y^{n} x^{n}, z\right]=0 .
\end{aligned}
$$

Then $R$ is comutative.

Throughout the paper $R$ denotes an associative ring $R$ and for all $x, y$ in $R,[x, y]=x y-y x$.

\section{The following lemma is due to Bell [4]:}

LEMMA 2.1. Let $R$ be a ring satisfying an identity $q(X)=0$, where $q(X)$ is a polynomial in a finite number of non-commuting indeterminates, its coefficients being integers with highest common factor 1. If there exists no prime $p$ for which the ring of $2 \times 2$ matrices over $G F(p)$ satisfies $q(X)=0$, then $R$ has a nilcommutator ideal and the nizpotent elements of $R$ form on ideal.

LEMMA 2.2. Let $R$ be a prime ring satisfying the hypothesis of the theorem. Then $R$ has no nonzero nilpotent element.

Proof. Let $x$ be an element of $R$ such that $x^{2}=0$. Using the hypothesis $\left(z_{1}\right)$ or $\left(z_{2}\right)$ of the Theorem, we get $(x y)^{n} z=z(x y)^{n}$, for all $y, z \in R$. With $z=x$, we get $(x y)^{n} x=0$, that is $(x y)^{n+1}=0$, for all $y$ in $R$. Whence it follows that $x R$ is a right ideal of $R$ in which $t^{n+1}=0$, for each $t \in x R$. Thus $x R=(0)$, by lemma 1.1 of [7]. This implies that $x=0$, since $R$ is prime.

Proof of the Theorem. We shall prove the result for rings satisfying $\left(z_{1}\right)$. In the other case one can get the result by proceeding on the same lines. Since $R$ is semi-prime satisfying the identity $q(x, y, z)=$ $(x y)^{n} z-x^{n} y^{n} z-z(x y)^{n}+z x^{n} y^{n}=0$, then it is isomorphic to a subdirectsum of prime rings $R_{\alpha}$ each of which as a homomorphic image of $R$ 
satisfies the hypothesis placed on $R$. Hence we can assume that $R$ is a prime ring satisfying $q(x, y, z)=(x y)^{n} z-x^{n} y^{n} z-z(x y)^{n}+z x^{n} y^{n}=0$, which is a polynomial identity with co-prime integral coefficients. Now if we consider $x=\left(\begin{array}{ll}0 & 1 \\ 0 & 0\end{array}\right), y=\left(\begin{array}{ll}0 & 0 \\ 1 & 0\end{array}\right)$ and $z=\left(\begin{array}{ll}0 & 1 \\ 0 & 0\end{array}\right)$, we find that no $2 \times 2$ matrix ring over $G F(p), p$ a prime, satisfies the identity. Hence by Lemma 2.1, $R$ has a nilcommutator ideal. But by Lemma 2.2, $R$ has no non-zero nilpotent elements. Thus the commutator ideal is zero and $R$ is commutative.

The following example shows that the above theorem is not true for arbitrary rings:

EXAMPLE. Let $D$ be a division ring and

$$
A_{K}=\left\{\left(a_{i j}\right) \in D_{K} \mid a_{i j}=O(i \geq j)\right\} \quad K>2 .
$$

Then $A_{3}$ is a noncommutative nilpotent ring of index 3 , which is not semi-prime, satisfies the identities in the hypothesis of the theorem.

\section{References}

[1] Hazar Abu-Khuzam, "Acommutativity theorem for rings", Math. Japon. 25 (1980), 593-595.

[2] -1 "A commutativity theorem for semi-prime rings", BulZ. Austral. Math. Soc. 27 (1983), 221-224.

[3] R. Awtar, "On the commutativity of non-associative rings", Publ. Math. Debrecen, 22 (1975), 177-188.

[4] H. E. Bell, "On some commutativity theorem of Herstein", Arch. Math. (Basel) 24 (1973), 34-38.

[5] -- "On the power map and ring commutativity", Canad. Math. BuZZ. 21 (1978), 399-404.

[6] I. N. Herstein, "Power Maps in rings", Michigan Math. J. 8 (1961), 29-32.

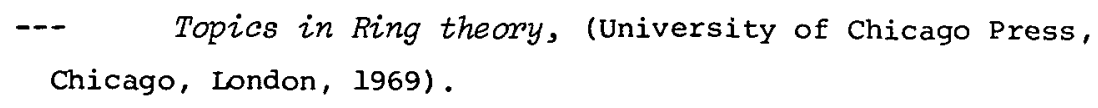

Department of Mathematics

Aligarh Muslim University

Aligarh 202001 (INDIA). 